\title{
FACTORES RELACIONADOS CON LA CONDUCTA ANTICONCEPTIVA EN MUJERES JÓVENES: ESTUDIO MIXTO
}

\section{FACTORS RELATED TO ANTI-CONCEPTIVE CONDUCT IN YOUNG WOMEN: MIXED STUDY}

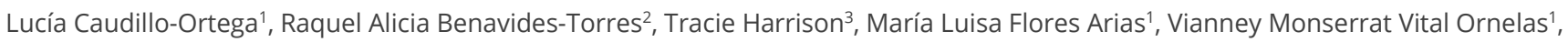
Carolina Valdez Montero 4

\author{
${ }^{1}$ Departamento de Enfermería sede Guanajuato, Universidad de Guanajuato, México \\ ${ }^{2}$ Facultad de Enfermería, Universidad Autónoma de Nuevo León, México \\ ${ }^{3}$ School of Nursing, University of Texas at Austin, USA
}

${ }^{4}$ Escuela Superior de Enfermería, Universidad Autónoma de Sinaloa, México

\section{Abstract}

Objective. To know the factors related to contraceptive behavior in Mexican women from 18 to 24 years old, using mixed methodology. Method. A transformative concurrent design was carried out, which allowed collecting and analyzing the quantitative and qualitative data simultaneously, then combining them and obtaining metainferences from the study. Results. The results were obtained from the combination of quantitative and qualitative data, with the average profile technique, the general average of the participants and of each of the nine interviewees of the qualitative phase were graphed. The narrative profiles were created to interpret the quantitative results, they were combined with the means of contraceptive behavior. It was found that the factors related to the contraceptive behavior of women were previous experience, attitudes, stigma, benefits, barriers, the support of the couple, the health personnel and the action plan that the woman has for the use of contraceptive methods. Conclusions. Women with high averages had a defined action plan for contraceptive behavior and women without a plan of action were more likely not to use contraception in their sexual relationships and to have an unplanned pregnancy.

Keywords: contraception, factors, mixed methods, women.

\section{Resumen}

Objetivo. Conocer los factores relacionados con la conducta anticonceptiva en mujeres mexicanas de 18 a 24 años, utilizando metodología mixta. Método. Se realizó un diseño concurrente transformativo, que permitió recolectar y analizar los datos cuantitativos y cualitativos simultáneamente, posteriormente combinarlos y obtener metainferencias del estudio. Resultados. Los resultados se obtuvieron de la combinación de los datos cuantitativos y cualitativos, con la técnica de perfil promedio, se graficó la media general de las participantes y de cada una de las nueve entrevistadas de la fase cualitativa. Se crearon los perfiles narrativos para interpretar los resultados cuantitativos, se combinaron con las medias de la conducta anticonceptiva. Se encontró que los factores relacionados con la conducta anticonceptiva de las mujeres fueron la experiencia previa, las actitudes, el estigma, los beneficios, las barreras, el apoyo de la pareja, del personal de salud y el plan de acción que tenga la mujer para el uso de métodos anticonceptivos. Conclusiones. Las mujeres con medias altas, tenían un plan de acción definido para la conducta anticonceptiva y las mujeres sin un plan de acción tenían mayor probabilidad de no usar métodos anticonceptivos en sus relaciones sexuales y presentar un embarazo no planeado.

Palabras clave: anticoncepción, factores, métodos mixtos, mujeres. 
El porcentaje de embarazos no planeados entre mujeres de 18 a 30 años es uno de los principales problemas de salud reproductiva a nivel mundial (Finner \& Henshaw, 2006; Nsubuga, Sekandi, Sempeera, \& Makumbi, 2016). Más del 50\% de los embarazos no planeados se reportan en mujeres menores de 29 años, principalmente por el bajo porcentaje en el uso de métodos anticonceptivos, derivado de conocimientos deficientes, falta de acceso a los servicios de planificación familiar y uso incorrecto. Los embarazos no planeados incrementan el riesgo de producir resultados maternofetales adversos, abortos en condiciones de riesgo y muertes maternas, principalmente en países en desarrollo (Ayoola, Nettleman, \& Brewer, 2007; Organización Naciones Unidas [ONU], 2012). La planificación familiar (PF) y la anticoncepción (AC) constituyen la base fundamental de la salud reproductiva (SR) por sus potenciales beneficios sociales y de salud (Organización Mundial de la Salud [OMS], 2017). La promoción del uso correcto de los métodos anticonceptivos contribuye a alcanzar el objetivo del milenio "mejorar la salud materna", con la prevención del $90 \%$ de los abortos en condiciones de riesgo, el $20 \%$ de la morbilidad relacionada al embarazo y el 32\% de las muertes maternas a nivel mundial (Cleland, Conde, Peterson, Ross, \& Tsui, 2012).

En México, la tasa más alta de fecundidad se reporta en el grupo de mujeres de 20 a 24 años de edad (28.5\%); la edad de inicio de la vida sexual activa es de 15 años. Asimismo, el $65.4 \%$ de las mujeres con vida sexual activa reportaron no usar ningún método anticonceptivo, lo que aumenta el riesgo de un embarazo no planeado así como de problemas de salud durante el embarazo, parto y puerperio (Instituto Nacional de Estadística Geografía e Informática [INEGI], 2014). En una encuesta reciente, se encontró que el método anticonceptivo más utilizado entre las mujeres jóvenes con vida sexual activa era el condón (84.5\%); además se reportó la disminución del uso de métodos hormonales en un 50\%. Con respecto a los embarazos no planeados, la encuesta reveló que los porcentajes más altos son en la población de adolescentes y las jóvenes (20 y 24 años), con 3.2 puntos y 2.4 puntos porcentuales, respectivamente (Consejo Nacional de Población [CONAPO], 2014).

La promoción de los métodos anticonceptivos (MA) como estrategia para reducir el número de embarazos no planeados ha sido empleada desde los años noventa. Sin embargo, el porcentaje de uso en mujeres jóvenes (20-24 años) continúa reportándose bajo (28.5\%); se trata de la población de mujeres menos estudiado en aspectos de reproducción y planificación familiar (Mendoza, Sánchez, \& Hernández, 2009). De acuerdo con las características sociodemográficas, las mujeres más vulnerables a una deficiente salud sexual y reproductiva, con reporte de menor uso de métodos anticonceptivos y poco acceso a los programas relacionados a la prevención de infecciones de transmisión sexual, como el VIH/Sida, son las mujeres solteras y jóvenes (18-24 años) que habitan en zonas de alta y muy alta marginación, zonas rurales e indígenas, con baja escolaridad y nivel socioeconómico bajo (Allen, Villalobos, Hernández, Suárez, De la Vara, \& De Castro, 2013).

La literatura también señala que existen múltiples factores que afectan el uso correcto y sostenido de los métodos anticonceptivos. Por la complejidad del fenómeno, se sabe que existen una serie de factores que influyen, como los motivos personales. El uso previo de algún método anticonceptivo como el condón o los métodos naturales (ritmo, billings) puede influir o no en la decisión de usar un MA: algunos estudios muestran que influye de forma positiva y otros que es de forma negativa, pero no existe evidencia determinante (Cox, Posner, \& Sangi, 2010; Ong, Smith, Wong, McNamee, \& Fairley, 2012; Wilson \& Koo, 2008).

Se ha encontrado que las mujeres tienen percepciones negativas sobre el uso de los anticonceptivos hormonales, entre las cuales se encuentran la insatisfacción con el método por los efectos secundarios (ganancia de peso, caída de cabello), el costo y la accesibilidad, entre otros. Sin embargo, estas percepciones pueden ser imaginarias (Ersek, Thompson, Huber, \& Findlow, 2010; Frost \& Darroch, 2008; Hooper, 2010; Littlejohn, 2012; Ushma \& Bennet, 2009). Por otra parte, también existen una serie de percepciones de tipo positivo por las cuales las mujeres desean usar MA efectivos como los anticonceptivos hormonales (AH), entre los que destaca el deseo de tener control sobre su vida, mayor estabilidad económica, emocional y lograr sus metas personales. No obstante, hay que destacar que no todas estas percepciones se han encontrado en los estudios (Bryant, 2009; Frost \& Darroch, 2008; Frost \& Lindberg, 2012; Hooper, 2010; Spies et al., 2010; Wu et al., 2010). 
Dentro de los factores socioculturales, las creencias religiosas son relevantes. Se ha reportado que las mujeres jóvenes tienden a negociar mayormente el uso de MA, mientras que las mujeres de mayor edad utilizan Ios MA naturales por considerar que no van en contra de las creencias religiosas. Los cambios en el sistema social influyen en los valores y los principios relacionados a la sexualidad y la reproducción, en especial al uso de los métodos anticonceptivos (Doctor, Phillips, \& Sakeah, 2009; Hirsch, 2009). El estatus socioeconómico influye en el uso y no uso, dado que se encuentra muy ligado a la educación, a la dependencia económica y la falta de empoderamiento para la toma de decisiones. Estudios realizados en poblaciones latinas indican que las mujeres con estudios e ingresos económicos limitados utilizan métodos efectivos, como las inyecciones o los implantes. Los métodos hormonales orales son más utilizados por mujeres de un nivel educativo y estatus socioeconómico más alto (Campo et al., 2012; Marston \& King, 2006). Otros estudios han encontrado que las mujeres de menor educación no utilizan ningún método por su falta de conocimiento del tema (Cox, Posner, \& Sangi, 2010; Frost \& Darroch, 2008; Kraft et al., 2010; Ong, Smith, Wong, McNamee, \& Fairley, 2012).

Las actitudes y las creencias positivas o negativas acerca de los MA se han asociado significativamente con el uso efectivo de los MA (Littlejohn, 2012; Rutherford, Anglemyer, Bagenda, Muyonga, Lindan, Barker, et al., 2014). Entre los factores interpersonales, es relevante el apoyo de la familia por ser la principal fuente de normas sociales y apoyo social para realizar conductas; también es determinante el papel de la pareja, al estar de acuerdo o en desacuerdo y aprobar el uso de MA (Cox, Posner, \& Sangi, 2010; Frost \& Lindberg, 2012; Glasier, 2010).

La anticoncepción es un fenómeno muy complejo que requiere un abordaje integral que pueda explicar la relación de las variables que influyen. Sin embargo, no se ha encontrado un modelo que incluya diversos factores en la conducta anticonceptiva en mujeres. En México, se han realizado investigaciones principalmente en adolescentes, pero son escasas en mujeres de 18 a 24 años; asimismo, los estudios abordan una o más variables y proporcionan una explicación parcial de la problemática. Por lo tanto, es importante cuestionarse acerca de los factores que intervienen en el uso de los MA.
La necesidad de evidencia científica que permita entender la problemática, clarificar por grupos de edad, nivel socioeconómico y en torno a las diferencias regionales, así como superar los retos en salud sexual y reproductiva que persisten, conduce a plantear el objetivo del estudio: conocer los factores relacionados con la conducta anticonceptiva (uso correcto y sostenido de los métodos anticonceptivos) en mujeres de 18 a 24 años de edad de una ciudad al noreste de México, a través de un diseño mixto.

\section{MÉTODO}

\section{Participantes}

Las participantes fueron elegidas bajo los siguientes criterios de inclusión: a) mujeres de 18 a 24 años de edad, b) que reportaron tener relaciones sexuales y utilizado algún método anticonceptivo alguna vez en su vida. Los criterios de exclusión fueron los siguientes: a) que la participante no fuera usuaria de un método anticonceptivo definitivo como oclusión tubárica bilateral o que su pareja tuviera vasectomía y b) mujeres con diagnóstico de esterilidad o infertilidad y participantes bajo tratamiento hormonal derivado de problemas de fertilidad (ver Figura 1).

El diseño de estudio fue de tipo mixto concurrente transformativo. El propósito de este diseño es hacer investigaciones orientadas al cambio de las realidades o transformación de metas de poblaciones consideradas como vulnerables o marginadas, donde la diferencia de poder es identificado (Creswell, 2008); en México, este desequilibrio puede intervenir en la sexualidad, reproducción y uso de anticonceptivos. El estudio fue realizado en dos fases simultáneas en recolección y análisis: la cuantitativa (CUAN) y la cualitativa (CUAL). Después del análisis se combinaron los resultados CUAN y CUAL y se obtuvieron las metainferencias del estudio; los apartados del trabajo se presentarán en este orden.

\section{Fase 1. Cuantitativa}

El diseño fue de tipo descriptivo, correlacional y transversal. La población fue de mujeres de 18 a 24 años de edad, quienes estaban inscritas y acudían como estudiantes a un instituto privado para el trabajo técnico en alguno de sus seis planteles al norte de México. El muestreo fue aleatorio, estratificado con asignación 
proporcional al tamaño del estrato; los estratos fueron cada uno de los seis planteles del instituto, ubicados en el área metropolitana, por lo que el número de participantes fue proporcional a la población total de cada plantel. Se generaron números aleatorios de las listas de asistencia sin sustitución para obtener a las participantes del estudio. Para calcular la muestra se utilizó el paquete estadístico n'Query Advisor, con un nivel de significancia de .05, un Coeficiente de Determinación de $r^{2}=.09$ y una potencia de prueba de $90 \%$ para un modelo de 13 variables, considerando una tasa de no respuesta del 30\%, con una muestra de 305 mujeres. Posteriormente, al verificar criterios de inclusión y exclusión, se descartaron 85 participantes.

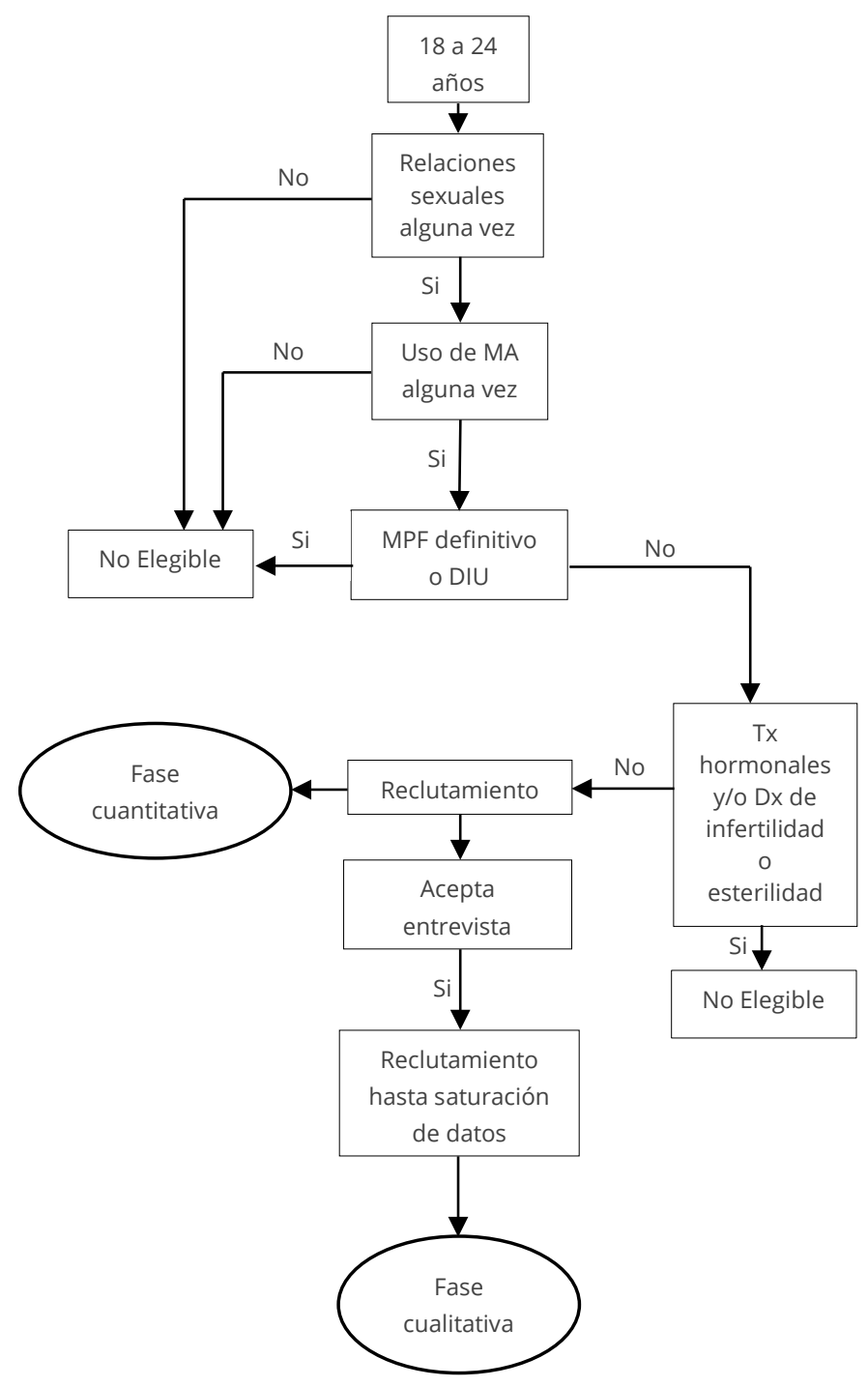

Figura 1. Diagrama de flujo de los criterios de elegibilidad

\section{Fase 2. Cualitativa}

El diseño fue una descripción cualitativa, con el propósito de describir situaciones y eventos; es decir, proporcionar información de cómo es y cómo se manifiesta determinado fenómeno. Se busca especificar las propiedades importantes de personas, grupos, comunidades o cualquier fenómeno que sea sometido a este tipo de análisis (Sandelowski, 2000). El muestreo fue por criterio lógico; implicó trabajar todos los casos que reunieran algún criterio predeterminado de importancia (uso de métodos anticonceptivos). Se entrevistaron mujeres que aceptaron participar voluntariamente en el estudio hasta la saturación de los datos. La recolección se detuvo en la entrevista nueve, con la que se logró la saturación. Se consideró saturación en el momento en que las entrevistas reflejaron una explicación relevante del tema; esta elección fue basada en lo que algunos investigadores refieren respecto a la saturación de los datos, enfatizando que ésta no se logra con la cantidad de información sino con la calidad de la misma.

Por su parte, Morse (1995) refiere que puede presentarse un falso sentido de saturación, el cual puede originarse por un enfoque demasiado restringido, una búsqueda mal encaminada o incluso una falta de perspicacia del investigador. Ella sugiere que la inclusión de nuevas unidades de observación debe realizarse hasta que el investigador disponga de los elementos necesarios para construir una teoría comprehensiva y convincente sobre el tema. También señala que lo importante no es el número de veces que aparece un dato, sino la detallada información de lo encontrado lo que dará su riqueza al estudio. Por su parte, Denzin (2010) menciona que la realidad en su complejidad no puede ser completamente capturada en ningún momento. No habría, entonces, punto de saturación. Ante esta declaración, Mayan (2009), menciona que el investigador debe continuar indagando no hasta el punto de una quizá inalcanzable saturación, sino hasta el momento en el que considere que puede decir algo importante y novedoso sobre el tema de estudio.

\section{Instrumentos}

Se aplicaron ocho instrumentos (ver Tabla 1) en formato de papel y lápiz, todos auto aplicables, con una duración para su llenado de una hora. Para la recolección de datos cualitativos, se realizó una entrevista semiestructuradas; la guía de entrevista contenía 
preguntas detonantes para que las participantes hablaran en profundidad de los temas (Mertens, 2005). Por lo tanto, la guía de entrevista se centró en obtener una descripción de la conducta anticonceptiva. Constaba de preguntas, las cuales permitieron obtener información acerca de las opiniones de las participantes, su percepción de los MA, experiencias con los MA, factores que influyeron en el uso de los mismos, motivación para usarlos y cambios en la vida de las mujeres con el uso de los MA, para completar la fase CUAN y CUAL simultáneamente.

Tabla 1. Descripción de los instrumentos

\begin{tabular}{|c|c|}
\hline Instrumento & Descripción \\
\hline Cédula de datos personales & $\begin{array}{l}\text { Aspectos sociodemográficos, uso } \\
\text { de métodos anticonceptivos, } \\
\text { historia sexual y reproductiva. }\end{array}$ \\
\hline $\begin{array}{l}\text { Actitudes para la conducta } \\
\text { anticonceptiva (Black \& Pollack, } \\
\text { 1987). }\end{array}$ & $\begin{array}{l}21 \text { ítems. Puntuaciones bajas } \\
\text { indican actitudes positivas y } \\
\text { puntuaciones altas indican } \\
\text { actitudes negativas. }\end{array}$ \\
\hline $\begin{array}{l}\text { Escala de compromiso religioso } \\
\text { (Worthington, McCullough, } \\
\text { Berry, \& Ripley, 2003). }\end{array}$ & $\begin{array}{l}10 \text { ítems. Puntuaciones van de } \\
10 \text { hasta 50, menor puntuación } \\
\text { menor compromiso con la } \\
\text { religión y a mayor puntuación } \\
\text { mayor compromiso con la } \\
\text { religión. }\end{array}$ \\
\hline $\begin{array}{l}\text { Beneficios y Barreras para la } \\
\text { conducta anticonceptiva } \\
\text { (Brunie, Tolley, Ngabo, Wesson, } \\
\text { \& Chen, 2013). }\end{array}$ & $\begin{array}{l}6 \text { ítems. Mide los aspectos } \\
\text { positivos y negativos de utilizar } \\
\text { los métodos anticonceptivos. }\end{array}$ \\
\hline $\begin{array}{l}\text { Autoeficacia para la conducta } \\
\text { anticonceptiva (Levinson, Wan, } \\
\text { \& Beame, 1998). }\end{array}$ & $\begin{array}{l}17 \text { ítems. A mayor puntuación } \\
\text { mayor capacidad percibida de la } \\
\text { mujer de que puede utilizar un } \\
\text { MA y viceversa. }\end{array}$ \\
\hline $\begin{array}{l}\text { Apoyo social para el uso de } \\
\text { métodos anticonceptivos } \\
\text { (Cutrona \& Russell, 1984; } \\
\text { Darbes \& Lewis, 2005). }\end{array}$ & $\begin{array}{l}72 \text { ítems. Mayor puntuación } \\
\text { mayor apoyo social. }\end{array}$ \\
\hline $\begin{array}{l}\text { Conducta anticonceptiva } \\
\text { (Jaccard, Dittus, \& Gordon, } \\
\text { 1996). }\end{array}$ & $\begin{array}{l}5 \text { ítems. Mayor puntación refiere } \\
\text { un mejor uso correcto y } \\
\text { sostenido de los métodos } \\
\text { anticonceptivos. }\end{array}$ \\
\hline Entrevista semiestructurada. & $\begin{array}{l}\text { Experiencias personales, } \\
\text { experiencias sociales, } \\
\text { experiencias interpersonales. }\end{array}$ \\
\hline
\end{tabular}

Fuente: Elaboración propia.

\section{Procedimiento}

El estudio fue aprobado por el Comité de Investigación y Ética de la Investigación de la Facultad de Enfermería de la Universidad Autónoma de Nuevo León. Se obtuvo el permiso correspondiente en la institución; a continuación, se solicitaron las listas de asistencia de cada plantel para obtener el total de la población asistente al instituto. Posteriormente, se capturaron los datos en un libro del programa Excel y se obtuvieron aleatoriamente las participantes del estudio. Después se acudió a la institución a invitar a las mujeres elegidas a participar en el estudio; se les informó que se estaría en un espacio destinado en el mismo plantel para tal propósito. Se acudió varios días al plantel designado en un horario accesible. Los días de la recolección de datos se llevaron los insumos necesarios (lápices, borradores) para el llenado de los instrumentos, los cuales fueron trasladados por el investigador en una caja cerrada.

Cuando las participantes se encontraban concentradas en el espacio designado, se les repitieron los objetivos, se les hizo énfasis en la confidencialidad y anonimato de sus respuestas; además, se les aseguró que podían retirarse del estudio sí ellas lo decidían. En primer lugar, se les proporcionó el consentimiento informado de la fase cuantitativa. Enseguida, la cédula de datos personales; ésta contenía dos preguntas filtro para verificar los criterios de inclusión. Si cumplía con estos se procedió a proporcionar los instrumentos para su llenado, con una duración aproximada de una hora.

Al mismo tiempo que la recolección de datos cuantitativos, se realizaron las entrevistas a las participantes que aceptaron voluntariamente y que además se consideraron sujetos representativos para el estudio, por su interacción con la conducta anticonceptiva. De forma individual se le explicó a cada una el objetivo de la entrevista, se les proporcionó el consentimiento informado, se realizó y grabó la entrevista con una hora aproximadamente de duración.

\section{Consideraciones Éticas}

El estudio se dirigió éticamente por la Ley General de Salud en materia de investigación para la salud (Secretaría de Salud [SSA], 1987), así como en la Declaración de Helsinki.

\section{Análisis de datos}

Para el análisis de datos se propusieron tres etapas para dar respuesta a los objetivos planteados, lograr la combinación de datos cuantitativos y cualitativos y obtener las metainferencias. En la etapa cuantitativa, los datos se analizaron utilizando el paquete estadístico Statistical Package for Social Sciences (SPSS) versión 19 para Windows. Se realizó la estadística descriptiva, a 
partir de la cual se obtuvieron frecuencias y porcentajes para las variables categóricas, así como medidas de tendencia central y dispersión para las variables numéricas, que sirvieron además para describir a la población de estudio. Para analizar las relaciones entre las variables, se realizaron correlaciones de Spearman y análisis de regresión lineal multivariado.

En la etapa cualitativa se realizó un análisis de contenido cualitativo, basado en las recomendaciones de Lincoln y Guba (1985). En la etapa mixta se combinaron los datos CUAN y CUAL (Creswell, 2009), a través de la técnicas de cuantificación de los datos cualitativos y la cualificación de los datos cuantitativos por la técnica del perfil promedio.

\section{RESULTADOS}

La media de edad de las participantes fue 20 años. Con respecto a su nivel educativo, reportaron de 9 a 14 años de estudio formales: la mayoría tenía solo la secundaria (50.5\%), seguido de preparatoria completa (24.1\%) y preparatoria trunca (22.3\%). El 68.6\% solo estudiaban, el 30\% estudiaban y tenían un empleo pagado y el $1.4 \%$ eran estudiantes y tenían un empleo no pagado. El $73.2 \%$ reportaron ser solteras, el $17.7 \%$ casadas y el $7.7 \%$ vivían en unión libre. El $78.2 \%$ de las participantes refirieron no tener hijos y el $21.8 \%$ tenían de uno a tres hijos.

\section{Historia sexual, reproductiva y anticonceptiva}

Con respecto a su primera menstruación, se obtuvo una media de 12 años; para el inicio de la vida sexual, se encontró una media de 16 años. Sobre su historia anticonceptiva, las participantes refirieron haber usado más de un MA previamente, entre los que destacan el condón masculino, los métodos naturales (ritmo y Billings), la anticoncepción de emergencia oral (AEO) y los anticonceptivos hormonales. El 53.6\% de las mujeres refirieron usar un método anticonceptivo y el $46.4 \%$ no utiliza ninguno; el método más utilizado fue el condón, seguido de los métodos naturales (ritmo y Billings), la AEO y los hormonales orales. Es importante destacar que la AEO se reporta con un consumo habitual; es decir, su uso es muy frecuente (5-6 veces por semana).

\section{Resultados Cuantitativos}

La Tabla 2 muestra los resultados de las asociaciones entre las variables del modelo cuantitativo y refleja que las actitudes para la conducta anticonceptiva (CA) se asocian con la conducta anticonceptiva $\left(F_{[4,215]}=\right.$ 12.155, $p<.001$ ), explicando la varianza en un 18.4\%; asimismo, los beneficios, las barreras y la autoeficacia que se asocian con la CA. Las variables explicaron la varianza en un $14.1 \%$ de la conducta anticonceptiva $(F$ $[4,215]=8.787, p<.001)$. Los resultados muestran que solo el apoyo de la pareja tiene efecto en la $C A\left(R^{2}=.08, F_{[4,215]}=\right.$ $17.14, p<.001)$.

Tabla 2. Resultados de la regresión lineal múltiple para la asociación de las variables

\begin{tabular}{|c|c|c|c|c|c|c|}
\hline Variable resultado & Parámetro & $B$ & $S E$ & $p$ & IC 95\% & $D E$ \\
\hline \multirow[t]{10}{*}{ Conducta anticonceptiva } & Edad & .02 & .04 & .577 & -.05 & .10 \\
\hline & Actitudes & -1.60 & .14 & .001 & -1.34 & -.79 \\
\hline & Compromiso con la religión & -.078 & .09 & .417 & -.26 & .10 \\
\hline & Ingreso económico & $-1.74 \mathrm{E}-05$ & $2.88 \mathrm{E}-05$ & .546 & $-8.01 E-05$ & 3.59E-05 \\
\hline & Beneficios & .17 & .07 & .020 & .041 & .33 \\
\hline & Barreras & -.33 & .10 & .002 & -5.41 & -.11 \\
\hline & Autoeficacia & .40 & .14 & .004 & .14 & .68 \\
\hline & Apoyo de la familia & -.11 & .11 & .289 & -.34 & .09 \\
\hline & Apoyo de la pareja & .95 & .11 & .001 & .60 & 1.31 \\
\hline & Apoyo del personal de salud & .16 & .18 & .372 & -.19 & .52 \\
\hline
\end{tabular}

Nota: Variable dependiente conducta anticonceptiva, $B=\mathrm{b}, \mathrm{SE}=$ Error estándar, $n=220$.

\section{Resultados Cualitativos}

Participaron nueve mujeres de 18 a 24 años; ninguna refirió estar casada o en unión libre. Tres tenían hijos y todas dependían económicamente de sus padres.
Del análisis cualitativo emergieron tres categorías con sus respectivas subcategorías (Tabla 3). 
Tabla 3. Categorías y subcategorías, análisis cualitativo

\begin{tabular}{ll}
\hline Categoría & Subcategorías \\
\hline Categoría 1: Motivos para usar un método anticonceptivo. Causas por las & Subcategoría 1. Preferencia y satisfacción con el método. \\
$\begin{array}{l}\text { cuales las mujeres usan un método anticonceptivo, que incluye aspectos } \\
\text { personales, interpersonales y socioeconómicos. }\end{array}$ & $\begin{array}{l}\text { Subcategoría 2. Miedo al embarazo. } \\
\text { Subcategoría 3. Tener planes para el futuro. } \\
\text { Subcategoría 4. Tiempo y tipo de relación con la pareja. }\end{array}$ \\
$\begin{array}{ll}\text { Categoría 2. Barreras para el uso de un método anticonceptivo. Causas por } \\
\text { las cuales las mujeres no usan un método anticonceptivo, que incluye solo } \\
\text { aspectos personales, ideas o pensamientos de las mujeres que las llevan a } \\
\text { no usar los métodos anticonceptivos. }\end{array}$ & $\begin{array}{l}\text { Subcategoría 1. Efectos de los métodos anticonceptivos. } \\
\text { Categoría 3. Apoyo/falta de apoyo de la red social. Percepción por parte de }\end{array}$ \\
$\begin{array}{l}\text { las mujeres de que su red social apoya o no el uso de los métodos } \\
\text { anticoncepticoncepción. } \\
\text { personal de salud. }\end{array}$ & $\begin{array}{l}\text { Subcategoría 2. Creencias negativas de los padres acerca de la sexualidad y } \\
\text { la reproducción. } \\
\text { Subcategoría 3. Apoyo de la pareja. }\end{array}$ \\
\end{tabular}

Fuente: Elaboración propia.

\section{Resultados mixtos}

El diseño concurrente transformativo tiene como finalidad converger la información cuantitativa y cualitativa, ya sea anidándola, conectándola o logrando su confluencia. Los datos recolectados por ambos métodos son comparados y/o mezclados en la fase de análisis (Hernández et al., 2005). En primer lugar, se realizó la técnica de cuantificación de las variables cualitativas (Tabla 4). Ésta consiste en transformar los datos cualitativos en datos numéricos. Las categorías y subcategorías de los datos cualitativos se transformaron en estadísticos descriptivos (frecuencias y porcentajes).

Cualificación de las variables cuantitativas: este proceso permite que los datos CUAN sean transformados en datos CUAL. La cualificación de los datos se utiliza para extraer más información de los datos cuantitativos o confirmar interpretaciones de los mismos. Existen cinco tipos de técnicas de cualificación: capital, comparativo, promedio y holístico. En el presente estudio se empleó la técnica de promedio, una descripción verbal de un grupo de participantes considerando la media de su atributo principal, con la finalidad de validar e interpretar los resultados CUAN. El atributo principal fue la conducta anticonceptiva (variable resultado). Ésta se midió con una escala tipo Likert que va de 0 a 4 puntos, en la que a mayor puntuación la conducta anticonceptiva es correcta y sostenida, mientras que a menor puntuación la conducta anticonceptiva es de riesgo. Esta técnica consta de tres pasos que se describen a continuación.

1. Se obtuvo la estadística descriptiva del atributo conducta anticonceptiva ( $\operatorname{Min}=0, \operatorname{Max}=4, M=1.8$, $D T=3.23)$. La media general se reportó baja; es decir, las mujeres utilizan los métodos anticonceptivos de forma incorrecta e inconsistente.

2. El objetivo del diseño mixto concurrente transformativo es la convergencia de los datos CUAN y CUAL, por la combinación de los mismos. Primero se obtuvieron las medias de las nueve participantes que participaron en la fase cualitativa $y$, derivado del análisis de los datos CUAL, se tomaron como conceptos clave motivos, barreras y apoyo/falta de apoyo de la red social para usar MA. Se desarrolló una metamatriz para organizar y depurar los datos. La metamatriz constó de la media reportada por las nueve participantes ( 0 a 3 ) y para interpretar esta puntuación promedio se sintetizaron las declaraciones que apoyaban el concepto de interés (Sandelowski, 2000; Tashakkori \& Teddlie, 2003). En la Tabla 4 se muestra la matriz simple que compara las puntuaciones y declaraciones de estos casos.

3. La comparación de los casos permitió conocer la convergencia de los datos y obtener las metainferencias de la conducta anticonceptiva de las mujeres (deducciones lógicas del tema).

En la metamatriz se observa que las mujeres que se encuentran en la puntuación mínima y debajo de la media son quienes reportan la conducta anticonceptiva de mayor riesgo. Las mujeres con puntuaciones por arriba de la media son quienes utilizan los MA de forma correcta y consistente. Para entender las puntuaciones y 
las declaraciones de las participantes, se presentan una serie de perfiles.

a) Las mujeres con puntuaciones debajo de la media reportan uno o más hijos, sin vida sexual activa, percepciones negativas y estigma hacia los MA y las mujeres que los usan. Su plan acerca de MA es no tener relaciones sexuales para evitar un embarazo no planeado; asimismo, sus experiencias con el personal de salud han sido poco favorables.

b) Las mujeres con un rango en la media de 2.0 a 2.2 reportan no tener hijos, son sexualmente activas, con abortos previos y no utilizan ningún tipo de método por no sentirse vulnerables a un embarazo, ni a las creencias negativas de los padres hacia la sexualidad, percepciones negativas de los MA o falta de apoyo de los padres. No consideran utilizar ningún tipo de método aunque tienen vida sexual activa.

c) Mujeres sin hijos, sexualmente activas: su objetivo del uso de MA es lograr sus planes y metas a futuro. Tanto el apoyo de la pareja para uso de MA como las creencias negativas de los padres acerca de la sexualidad, y los MA, promueven en las mujeres conductas menos riesgosas, así como evitar un embarazo, continuar con el apoyo económico y facilitar el logro de metas.

Tabla 4. Metamatriz, convergencia de datos cuantitativos y cualitativos

\begin{tabular}{lcc}
\hline Subcategoría & Frecuencia & $\%$ \\
\hline Tener planes para el futuro & 4 & 44.4 \\
Efectos de los métodos anticonceptivos & 4 & 44.4 \\
Estigma hacia la anticoncepción & 5 & 55.5 \\
Miedo al embarazo & 5 & 55.5 \\
Tiempo y tipo de relación & 5 & 55.5 \\
Déficit de conocimientos & 5 & 55.5 \\
Creencias negativas de los padres hacia la & 5 & 55.5 \\
sexualidad y la reproducción & & \\
No apoyo del personal de salud & 5 & 55.5 \\
No apoyo de los padres & 6 & 66.6 \\
Apoyo de la pareja & 6 & 66.6 \\
Preferencia y satisfacción del método & 7 & 77.7 \\
\hline
\end{tabular}

Fuente: Elaboración propia.

Emergieron cuatro metainferencias de la combinación y análisis de los datos CUAN y CUAL: plan de acción, estigma hacia la anticoncepción, creencias de los padres acerca de la sexualidad y la reproducción y los proveedores de salud.

\section{Plan de acción}

El plan de acción se refiere a la identificación de estrategias que deben emplearse para evitar conductas de riesgo; están influenciadas por el contexto, así como por los aspectos psicológicos, socioculturales e interpersonales. Las mujeres que no cuentan con estrategias para el uso efectivo de los métodos anticonceptivos en diversas situaciones se encuentran en mayor riesgo de presentar uso incorrecto de los MA.

\section{Estigma hacia la anticoncepción}

La atribución de características negativas hacia los MA, en especial los hormonales por considerar que no son socialmente aceptables, contribuyen en mantener un estigma. Asimismo, la sociedad estigmatiza a las mujeres que usan los métodos anticonceptivos por considerar esta conducta contra las normas y patrones morales establecidos.

Creencias de los padres acerca de la sexualidad y la reproducción

Las creencias de los padres acerca de la sexualidad y la reproducción tienen una influencia importante en el uso y no uso de los MA, dado que en el contexto de algunas familias mexicanas los temas acerca de la sexualidad son considerados como tabúes y no se permite hablar abiertamente de ellos. Es el caso de la menstruación, las relaciones sexuales prematrimoniales y el uso de MA, entre otros. Estas creencias disminuyen la comunicación con los padres sobre temas de sexualidad y reproducción.

Proveedores de salud

El personal de salud (médicos, enfermería, psicólogos, entre otros) desempeña un papel fundamental en la atención de las mujeres en edad reproductiva. Sin embargo, algunas actitudes o conductas pueden generar que las mujeres perciban poco apoyo (falta de información) reproductivo.

El modelo final muestra la combinación de las variables CUAN y CUAL que proporcionan una serie de factores que influyen en la conducta anticonceptiva de las mujeres jóvenes (Figura 3). 
Características

individuales y

experiencias

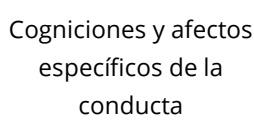

Resultado

conductual

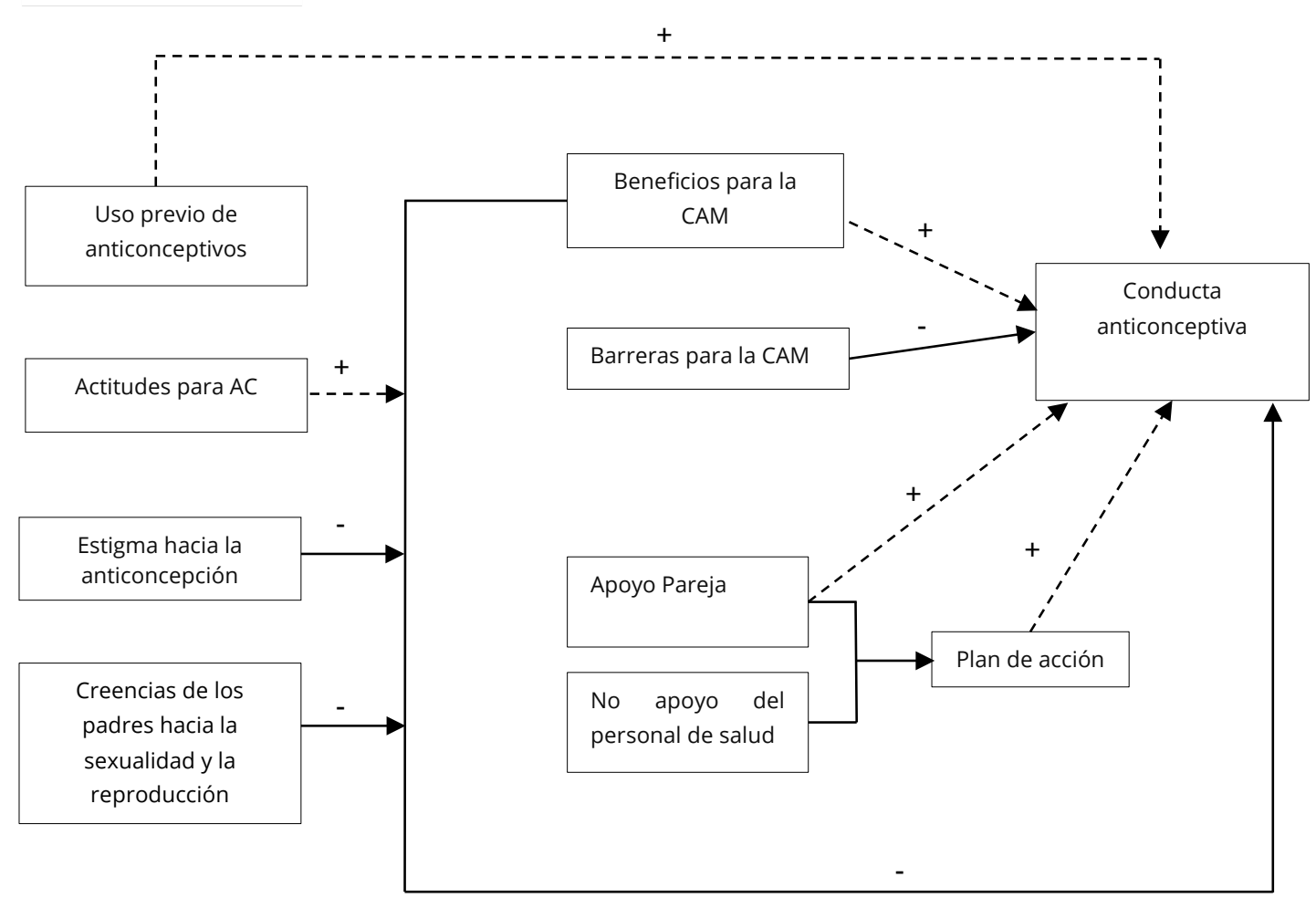

Figura 2. Modelo de la conducta anticonceptiva en mujeres (MOCAM)

\section{DISCUSIÓN}

Este estudio tuvo como objetivo describir los factores relacionados con la conducta anticonceptiva (uso correcto y sostenido de los métodos anticonceptivos) de las mujeres de 18 a 24 años de edad de una ciudad al noreste de México, utilizando metodología mixta. Ésta resultó especialmente acertada para abordar el fenómeno de la conducta anticonceptiva en mujeres, dado que el análisis se realiza desde varias perspectivas y permite la integración de nuevas variables derivadas de la combinación de los datos CUAN y CUAL. Asimismo, la metodología cuantitativa permitió un abordaje general de la conducta anticonceptiva y la metodología cualitativa sirvió para conocer las percepciones y describir el fenómeno; también se considera una metodología innovadora que permitió describir profunda y ampliamente el fenómeno.

A pesar de que la salud materna es un objetivo del milenio, continúa existiendo al respecto una desigualdad en muchos países, principalmente en los que se encuentran en desarrollo. En México, el porcentaje de embarazos no planeados es elevado, así como en otros países de Latinoamérica, de acuerdo a lo reportado en estadísticas internacionales. Por otra parte, un alto porcentaje de mujeres refieren estar sexualmente activas en comparación a lo reportado por el Consejo Nacional de Población (CONAPO, 2014): 52.9\% contra 65\% del presente estudio; esto es debido a que el inicio de las relaciones sexuales es a más temprana edad.

Con respecto a las actitudes, las mujeres que las reportaron positivas se percibían con mayor capacidad de utilizar los MA de forma correcta y sostenida; esto es similar a lo reportado por otros autores (Nsubuga, Sekandi, Sempeera, \& Makumbi, 2016; Sieving \& Bearinger, 2007), donde las mujeres con actitudes positivas se sentían con mayor capacidad de usar un método de forma correcta, como las pastillas o las inyecciones anticonceptivas.

Los beneficios y las barreras para la conducta anticonceptiva influyen en las mujeres. Al percibir un mayor número de consecuencias positivas al usar los métodos anticonceptivos, las mujeres que los emplean son constantes con su uso; lo anterior es consistente con 
los reportes de algunos autores (Frost \& Lindberg, 2012; Ntata, Mvula, \& Adamson, 2012). Las barreras percibidas en mayor proporción como el costo, los efectos adversos $y$ el temor a no poderse embarazar en un futuro, incrementan el riesgo de no usarlo o abandonarlos; esto es similar a lo reportado por otros autores (Frost \& Lindberg, 2012; Littlejohn, 2012).

El apoyo de la pareja es un factor muy importante para la conducta anticonceptiva: diversos autores lo mencionan como un predictor de la conducta anticonceptiva (Kenyon, Sieving, Jerstad, Pettingell, \& Skay, 2010; Ong, et al., 2012). El estigma hacia los MA influye de forma negativa, en especial hacia aquellos de tipo hormonal por considerarlos en contra de la naturaleza humana; además, existe el estigma hacia las personas que utilizan un método anticonceptivo debido a que va en contra de sus normas culturales o de los patrones establecidos dentro de la sociedad. Algunos estudios concuerdan con estos hallazgos (Egede, et al., 2015; Melaku, Berhane, Kinsman, \& Hailemariam, 2014).

En relación a las creencias de los padres acerca de la sexualidad y la reproducción, éstas tienen sus raíces en la cultura y la religión. La mujer es considerada tradicionalmente como secundaria e inferior al hombre; ha sido víctima de una sociedad patriarcal que "se ha encargado de idealizar a un hombre y a una mujer que responden a patrones previstos, asignados a uno y otro sexo por culturas, religiones y tradiciones" (Hill, Siwatu, \& Robinson, 2013).

El personal de salud que tiene relación con las mujeres en el aspecto de sexualidad y reproducción al proporcionar información objetiva y orientación oportuna para la toma de decisiones, incrementa la probabilidad de que las mujeres usen MA. Sin embargo, el personal que actúa de forma sobreprotectora o con actitudes negativas contribuye al abandono de los métodos, como lo reporta Brunie (2003). En este sentido, la OMS ha identificado un problema creciente que afecta la salud de las mujeres: "la anticoncepción forzada". Este acto implica presionar a las mujeres a utilizar un MA o administrarlo sin consentimiento (OMS, 2014). Es una clara violación al derecho a la información de las personas asentado en tratados internacionales, como la Convención Americana sobre Derechos Humanos. Para que este derecho se cumpla no debe existir violación alguna sobre cualquier mujer; las personas deben tener acceso a información veraz, objetiva, imparcial y libre de prejuicios acerca de las opciones anticonceptivas que tienen. Además, esta información debe ser proporcionada en un lenguaje adecuado y comprensible, así como en un ambiente libre de presiones y coerción, con el objetivo que las mujeres decidan libremente otorgar o no su consentimiento para recibir un MA (Organización de los Estados Americanos [OEA], 1969).

En el estudio se presentan casos donde se informa algún tipo de anticoncepción forzada; éstos no deben ser considerados como hechos aislados, sino como la manifestación de un problema creciente que se reporta en forma escasa y al cual no se le da seguimiento. Es importante sensibilizar al personal de salud que se encuentra en contacto con las usuarias de MA y evitar la normalización de estos actos.

Los resultados de tipo mixto permitieron combinar los resultados e incorporar variables al modelo. El plan de acción, tal y como define Pender (2006), implica seguir el siguiente proceso cognitivo: 1) el compromiso es llevar a cabo una acción específica en un tiempo y lugar determinado, con personas específicas o la persona sola, independientemente de sus preferencias; 2) identificación de estrategias que lleguen a producir, llevar a cabo o reforzar una conducta. En este estudio, solo algunas de las mujeres llegan a formular estrategias bien establecidas como el uso de métodos efectivos, aunque también existen mujeres que no tienen plan para el uso de MA.

Los hallazgos del estudio brindan una imagen del fenómeno de la conducta anticonceptiva en mujeres. La metodología mixta fue especialmente acertada para abordarlo y el diseño concurrente transformativo es innovador y está enfocado en el cambio de las realidades de la población en estudio en desbalance de poder.

\section{Conclusiones}

Con base en los resultados, se puede concluir que la edad promedio en que las mujeres inician su vida sexual es de 16 años, similar a la reportada en la media nacional. El uso previo de anticonceptivos es una variable muy importante: representa la experiencia previa para reforzar una conducta, por lo que el uso previo de $A C$ influye en que una mujer utilice de forma correcta y sostenida métodos anticonceptivos posteriormente. Las actitudes son una variable importante, debido a que, si estas son positivas, las mujeres perciben un mayor 
número de beneficios al usarlos y son quienes tienen un uso correcto y sostenido de los mismos.

Las mujeres que perciben un mayor número de consecuencias positivas al usar los métodos anticonceptivos presentan un uso correcto y sostenido de los métodos anticonceptivos. Las mujeres que se perciben con una capacidad alta para utilizar los métodos anticonceptivos tienden a ser usuarias consistentes. Cuando las mujeres perciben apoyo por parte de su pareja para utilizar métodos anticonceptivos manifiestan un uso correcto y sostenido de los métodos anticonceptivos.

Con respecto a los resultados cualitativos, las categorías y subcategorías que emergieron permitieron vislumbrar una imagen descriptiva del fenómeno de la anticoncepción en el grupo de edad de 18 a 24 años. Se reportaron barreras para utilizar métodos anticonceptivos: los efectos de los métodos anticonceptivos o las consecuencias derivadas de su uso, en especial de los hormonales y del dispositivo intrauterino (DIU). Por otra parte, el estigma hacia los métodos anticonceptivos y quienes los usan influye de forma negativa en las mujeres para usar un método anticonceptivo. Dicho estigma se presenta especialmente alrededor de los métodos que contienen hormonas, adjudicándoles efectos extravagantes. También se encuentra el estigma hacia las mujeres que los usan, por considerar que su comportamiento es en contra de las normas culturales establecidas, debido a que su utilización de un método anticonceptivo se relaciona con que tienen múltiples parejas sexuales; es un estigma social fundado en preceptos religiosos arraigados y transmitidos de generación en generación.

En relación al apoyo de otras personas, las mujeres perciben apoyo de la pareja para utilizar métodos anticonceptivos. Por otra parte, la falta de apoyo de los padres para el uso de los métodos anticonceptivos está muy relacionado con las creencias acerca de la sexualidad y la reproducción. Los padres no aceptan las relaciones prematrimoniales ni el uso de métodos anticonceptivos, por no ser social, cultural y religiosamente aceptable. Las mujeres sienten que el personal de salud no les apoya para el uso de MA; en algunos casos no se proporciona información veraz y objetiva, ni orientación oportuna sobre las opciones anticonceptivas que existen. Al contrario, perciben presión para usar un MA. Esto disminuye la probabilidad del uso correcto del mismo. La escasa información acerca de la anticoncepción forzada en países latinoamericanos permite que se sugiera abordar este tema en los diferentes contextos y poder generar líneas de acción en los diferentes niveles de atención a la salud.

\section{Limitaciones}

El diseño de estudio fue de tipo concurrente transformativo, sin embargo la recolección simultánea de los datos tuvo un desfase de varios días, por el tiempo de las participantes para las entrevistas de la fase cualitativa no. Referente al análisis de los datos, la principal limitación fue la escasa literatura de estudios con un diseño concurrente transformativo, así como pocos investigadores capacitados en esta metodología.

Con respecto a la teoría utilizada Modelo de Promoción de la Salud, fue apropiada sin embargo, referente a los factores personales psicológicos y socioculturales el modelo no define concretamente los conceptos y los alcances de estos, solo se mencionan algunos ejemplos, pero no queda claro si casos de interculturalidad, indigenismo migración pudieran ser explicados con este modelo. En relación a los instrumentos empleados, una de las limitaciones fue el tiempo prolongado de aplicación. La escala de provisión social para la conducta anticonceptiva en mujeres, era muy extenso para las participantes se sugiere revisar una versión corta.

\section{REFERENCIAS}

Allen, B., Villalobos, A., Hernández, M. I., Suárez, L., De la Vara, E., \& De Castro, F. (2013). Inicio de vida sexual, uso de anticonceptivos y planificación familiar en mujeres adolescentes y adultas en México, Salud Publica México, 55(2), 235-240.

Ayoola, A. B., Nettleman, M., \& Brewer, J. (2007). Reasons for unprotected intercourse in adult women. Journal of Women's Health, 16 (3), 302 310.

Black, K. J. \& Pollack, R. H. (1987). The development of a contraceptive attitude scale. Paper presented at the Annual meeting of the Southern Society of Philosophy and Psychology; Atlanta.

Brunie, A., Tolley, E., Ngabo, F., Wesson, J., \& Chen, M. (2013). Getting to 70\%: Barriers to modern contraceptive use for women in Rwanda. International Journal of Gynecology and Obstetrics, 123 (1), 11-15. doi: 10.1016/j.ijgo.2013.07.005

Cleland, J., Conde, A., Peterson, H., Ross, J., \& Tsui, A. (2012). Contraception and health. Lancet, 380, 149-56. 
Consejo Nacional de Población. Estrategia Nacional para la Prevención de Embarazos en Adolescentes. 2014. Recuperado en enero 2015 desde:

http://www.conapo.gob.mx/work/models/CONAPO/Resource/244 1/1/images/ENAPEA V10.pdf

Cox, S., Posner, S., \& Sangi, H. (2010). Who's Responsible? Correlates of Partner Involvement in Contraceptive Decision Making. Women's Health, 20, 254-259.

Creswell, J. W. (2009). Research design: Qualitative, Quantitative and mixed methods approaches. (Third Ed). Los Angeles: SAGE.

Cutrona C. E., \& Russell D. W. (1987). The provisions of social relationships and adaptations to stress. En W. H. Jones \& D. Perlman (Eds.) Advances in personal relationships, (pp. 37-67). Greenwich: JAI Press.

Darbes L. A. \& Lewis. M. A. (2005). HIV-specific social support predicts less sexual risk behavior in gay male couples. Health Psychology, 24(6), 617-22.

Denzin, N. (2010). Moments, Mixed Methods, and Paradigm Dialogs. Qualitative Inquiry, 16(6), 419-427.

Egede, J. O., Onoh, R. C., Umeora, O. U., lyoke, C. A., Dimejesi, I. B., \& Lawani, L. O. (2015). Contraceptive prevalence and preference in a cohort of South-East Nigerian women. Dove Press, 9, 707-714.

Finner, L. B., \& Henshaw, S.K. (2006). Disparities in Rates of Unintended Pregnancy in the United States, 1994 and 2001. Perspectives on Sexual and Reproductive Health, 38(2), 90-96.

Frost, J., \& Lindberg, L. (2012). Reasons for using contraception: perspectives of US women seeking care at specialized family planning clinics. Contraception, 87, 465-472.

Hill, N. J., Siwatu, M., \& Robinson, A. (2013). "My Religion Picked My Birth Control": The Influence of Religion on Contraceptive Use. Journal of Religion Health.

Instituto Nacional de Estadística y Geografía. Encuesta Nacional de Dinámica Demográfica. 2014. Recuperado en abril, 2015 desde: http://www.beta.inegi.org.mx/proyectos/enchogares/especiales/e nadid/2014/

Jaccard, J., Dittus, P. J., \& Gordon, V. V. (1996). Maternal correlates of adolescent sexual and contraceptive behavior. Family Planning Perspectives, 28, 159-165.

Kenyon, D. B., Sieving, R. E., Jerstad, S. J., Pettingell, S. L., \& Skay, C. (2010). Individual, interpersonal, and relationship factors predicting hormonal and condom use consistency among adolescent girls. Journal Pediatric Health Care, 24, 241-249.

Kornides, M. L., Kitsantas, P., Lindley, L. L., Wu, H. (2015). Factors Associated with Young Adults' Pregnancy Likelihood. Journal midwifery women's health, 60(2), 158-168.

Levinson, R., Wan, C., \& Beame, L. (1998). The Contraceptive Self-Efficacy Scale: Analysis in Four Samples. Journal of Youth and Adolescence, 27 (6).

Lincoln, Y. S. \& Guba, E. G. (1985). Naturalistic Inquiry. London: Sage.
Littlejohn, K. E. (2012). Hormonal Contraceptive Use and Discontinuation Because of Dissatisfaction: Differences by Race and Education Demography, 49, 1433-1452.

Mayan, M. (2009). Essentials of qualitative inquiry. Walnut Creek: Left Coast Press, Inc.

Melaku, Y. A., Berhane, Y., Kinsman, J., \& Hailemariam, L. R. (2014). Sexual and reproductive health communication and awareness of contraceptive methods among secondary school female students, northern Ethiopia: a cross-sectional study. Biomedic Central Public Health, 14, 252.

Mendoza, D., Sánchez, M., Hernández, M. F., \& Mendoza, M. E. (2009). 35 años de planificación familiar en México. La situación demográfica de México. México: Consejo Nacional de Población.

Morse, J. (1995). The significance of saturation. Quality Health Research Journal, 5(2), 147-149.

Nsubuga, H., Sekandi, J., Sempeera, H., \& Makumbi, F. (2016). Contraceptive use, knowledge, attitude, perceptions and sexual behavior among female University students in Uganda: a crosssectional survey. BMC Women's Health, 16 (6), 1-11. doi: 10.1186/s12905-016-0286-6

Ntata, P., Mvula, P., \& Adamson, S. M. (2012). “Condoms make you lose both the child and pleasure": perceptions on contraceptives use in Malawi. Tanzania Journal of Health Research, 15(1), 1-12. doi: 10.4314/thrb.v15i1.1

Ong, J., Smith, M., Wong, W., McNamee, K., \& Fairley, C. (2012). Contraception matters: indicators of poor usage of contraception in sexually active women attending family planning clinics in Victoria, Australia. Biomedic Central Public Health, 12, 1108. doi: 10.1186/1471-2458-12-1108

Organización de las Naciones Unidas. Objetivos de Desarrollo del Milenio Informe. 2012. Recuperado en marzo 2014 desde : http://www.un.org/es/millenniumgoals/pdf/mdg-report-2013spanish.pdf

Organización de los Estados Americanos. (2014). Convención Americana sobre derechos humanos (Pacto de San José). Recuperado en octubre 2018 desde: https://www.oas.org/dil/esp/tratados B32 Convencion Americana sobre Derechos Humanos.pdf

Organización Mundial de la Salud. (2016). Planificación familiar: Centro de prensa. Recuperado en junio 2016 desde: http://www.who.int/mediacentre/factsheets/fs351/es/

Organización Mundial de la Salud (2014). Respeto de los derechos humanos al proporcionar información y servicios de anticoncepción: orientaciones y recomendaciones. Recuperado en octubre 2018 desde: http://www.who.int/topics/human rights/derechos humanos anti concepcion spa.pdf

Pender, N., J. (2006). Health promotion in nursing Practice. Third edition. London: Appleton \& Lange.

Rutherford, G., W., Anglemyer, A., Bagenda, D., Muyonga, M., Lindan, C.P., Barker, J.L. et al. (2014). University students and the risk of HIV and 
other sexually transmitted infections in Uganda: the Crane survey. International Journal Adolescent Medicine Health, 26(2), 209-215.

Sandelowski, M. (2000). Focus on Research Methods Whatever Happened to Qualitative Description? Research in Nursing \& Health, 23, 334-340.

Sieving, R. E., \& Bearinger, L. H. (2007). Adolescent Dual Method Use: Relevant Attitudes, Normative Beliefs and Self-Efficacy. Journal Adolescent Health, 40(3), 275-275.

Worthington, E., McCullough, M., Berry, J., \& Ripley, J. (2003). The Religious Commitment Inventory-10: Development, refinement, and validation of a brief scale for Research and Counseling. Journal of Counseling Psychology, 50(1), 84-96. 